\title{
New $\mathrm{Sb}_{2} \mathrm{Te}_{3-x} \mathrm{Se}_{x}$ Monolayers with High
}

\section{Electron Mobilities and Wide Absorption}

\section{Range}

\section{Supplementary}

Ying Chen, ${ }^{\dagger} \mathrm{Yu} \mathrm{Wu},{ }^{\ddagger} \mathrm{Ke} \mathrm{Xu},{ }^{\ddagger}$ Congcong $\mathrm{Ma},{ }^{\dagger}$ Zixuan Lu${ }^{\dagger}$ Xintong Zhang, ${ }^{\ddagger}$ Hao Zhang, ${ }^{*,+}, \mathbb{1}$ Heyuan Zhu, ${ }^{\ddagger}$ and Zhilai Fang ${ }^{*, \dagger}$

$\dagger$ Academy for Engineering and Technology, Fudan University, and Engineering Research Center of Advanced Lighting Technology, Ministry of Education, Shanghai 200433, China $\ddagger$ Key Laboratory of Micro and Nano Photonic Structures (MOE) and Department of Optical Science and Engineering, Fudan University, Shanghai 200433, China \Nanjing University, National Laboratory of Solid State Microstructure, Nanjing 210093, China.

E-mail: zhangh@fudan.edu.cn; zlfang@fudan.edu.cn

\section{$1 \quad a b$ initio molecule dynamics (AIMD) simulation}

We also carry out the ab initio molecule dynamics (AIMD) simulation to study the thermal stability of $\mathrm{Sb}_{2} \mathrm{Te}_{3-x} \mathrm{Se}_{x}$ monolayers at high temperatures. The AIMD simulation results shown in Fig. S1(a-i) verify that $\alpha-\mathrm{Sb}_{2} \mathrm{TeSe}_{2}$ monolayer remains stable up to at least $700 \mathrm{~K}$, $\alpha-\mathrm{Sb}_{2} \mathrm{Te}_{2} \mathrm{Se}$ and $\beta-\mathrm{Sb}_{2} \mathrm{TeSe}_{2}$ monolayers remain stable up to at least $900 \mathrm{~K}$. 

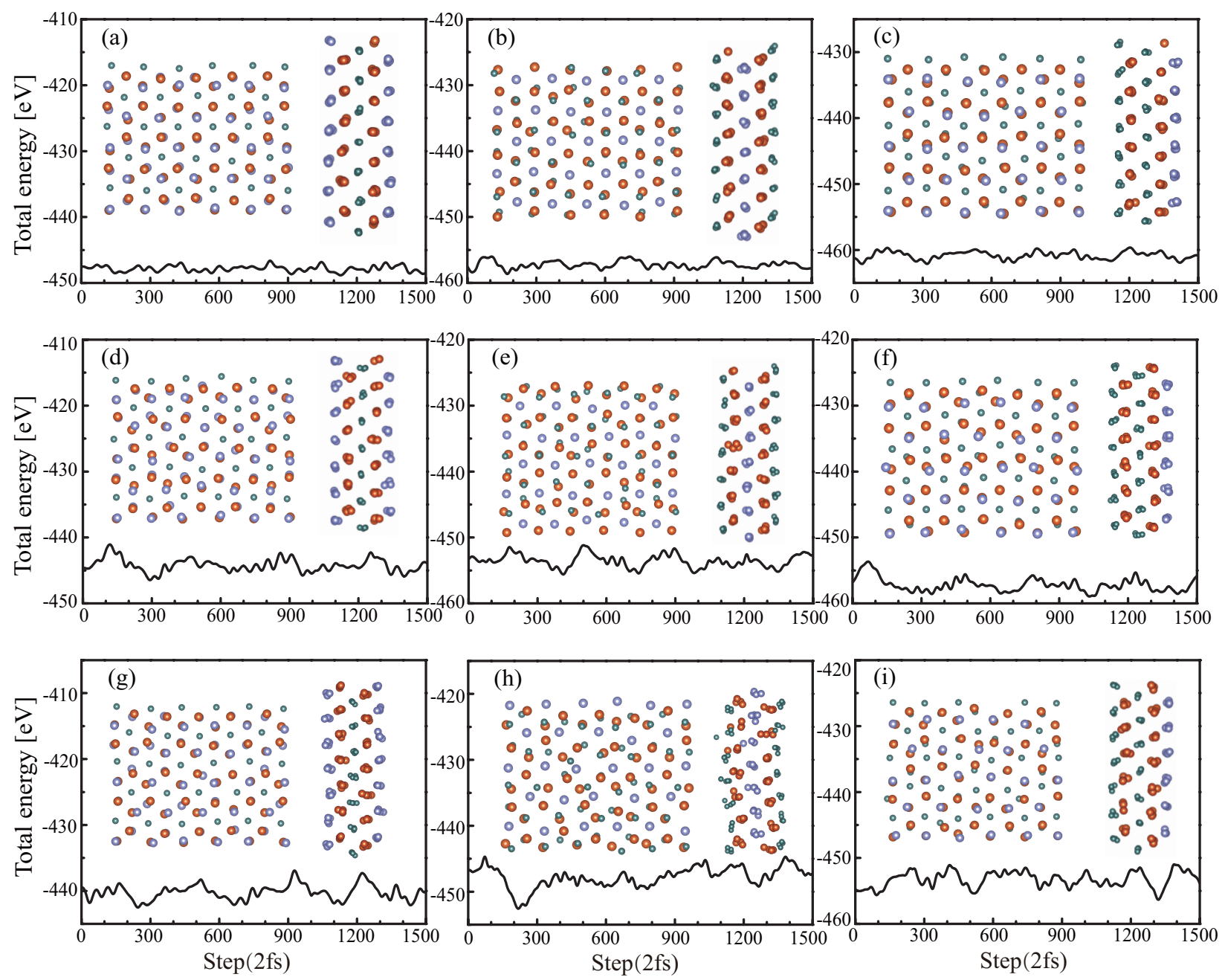

Figure S1: The AIMD calculation results of $(\mathrm{a} / \mathrm{d} / \mathrm{g}) \alpha-\mathrm{Sb}_{2} \mathrm{Te}_{2} \mathrm{Se},(\mathrm{b} / \mathrm{e} / \mathrm{h}) \alpha-\mathrm{Sb}_{2} \mathrm{TeSe}_{2}$ and (c/f/i) $\beta-\mathrm{Sb}_{2} \mathrm{TeSe}_{2}$ at $500 / / 700 / 900 \mathrm{~K}$.

\section{Electronic structure}

Fig. S2(a,b,c) shows the band structures of $\mathrm{Sb}_{2} \mathrm{Te}_{3-x} \mathrm{Se}_{x}$ monolayers calculated by PBE method, bandgaps of $\alpha-\mathrm{Sb}_{2} \mathrm{Te}_{2} \mathrm{Se} / \alpha-\mathrm{Sb}_{2} \mathrm{TeSe}_{2} / \beta-\mathrm{Sb}_{2} \mathrm{TeSe}_{2}$ are 0.63/0.75/0.69 eV. Considering the effect of SOC, bandgaps of $\alpha-\mathrm{Sb}_{2} \mathrm{Te}_{2} \mathrm{Se} / \alpha-\mathrm{Sb}_{2} \mathrm{TeSe}_{2} / \beta-\mathrm{Sb}_{2} \mathrm{TeSe}_{2}$ are $0.44 / 0.61 / 0.53$ $\mathrm{eV}$ [shown in Fig. S2(d,e,f)]. Fig. S2(g,h,i) shows band structures of $\mathrm{Sb}_{2} \mathrm{Te}_{3-x} \mathrm{Se}_{x}$ monolayers calculated by HSE method, and bandgaps of $\alpha-\mathrm{Sb}_{2} \mathrm{Te}_{2} \mathrm{Se} / \alpha-\mathrm{Sb}_{2} \mathrm{TeSe}_{2} / \beta-\mathrm{Sb}_{2} \mathrm{TeSe}_{2}$ are 1.04/1.24/1.14 eV. In all the calculation results of band structures, the three $\mathrm{Sb}_{2} \mathrm{Te}_{3-x} \mathrm{Se}_{x}$ monolayers are indirect-bandgap semiconductors. Although the bandgaps calculated by 

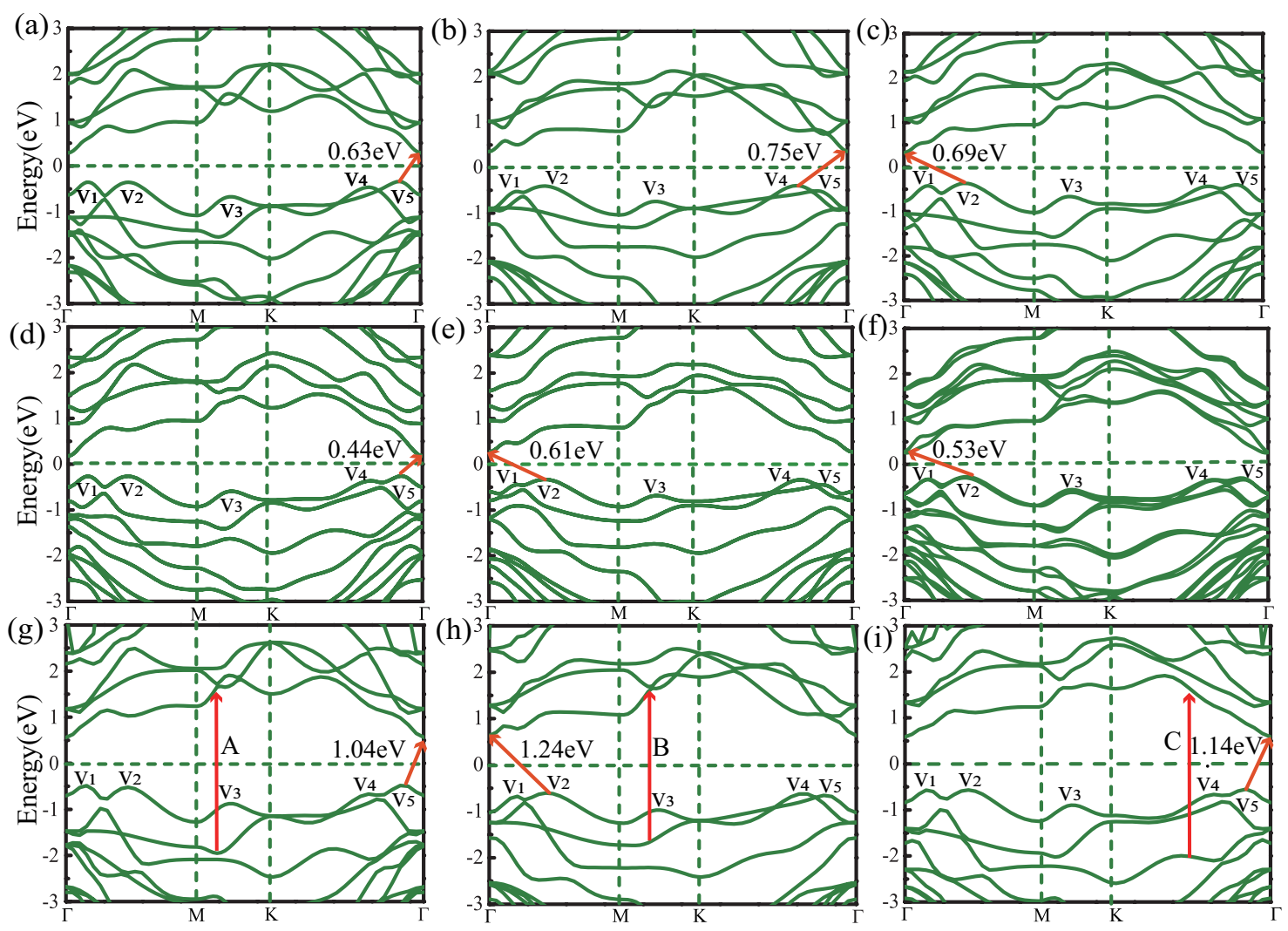

Figure S2: The PBE, PBE+SOC, HSE band structure of $(\mathrm{a}, \mathrm{d}, \mathrm{g}) \alpha-\mathrm{Sb}_{2} \mathrm{Te}_{2} \mathrm{Se},(\mathrm{b}, \mathrm{e}, \mathrm{h}) \alpha-$ $\mathrm{Sb}_{2} \mathrm{TeSe}_{2}$ and (c,f,i) $\beta-\mathrm{Sb}_{2} \mathrm{TeSe}_{2}$.

PBE (with SOC)/HSE are smaller/larger than the bandgaps calculated by PBE (without SOC), the overall shapes of the bands remain unchanged, which means that we can still calculate their electrical transport properties using PBE method.

In order to quantitatively describe the chemical bonds for crystals, Bloechl et al proposed the crystal orbital Hamiton population (COHP) method based on the partitioning of the band structure energy into bonding, nonbonding and antibonding contributions. ${ }^{1}$ The criterion for bonding and antibonding states is characterized by a negative/positive Hamiltonian off-site element given by $H_{\mu v}=\langle\mu|H| v>$, in which $| \mu>$ and $\mid v>$ are adjacent orbitals. As shown in Fig. S3, we also use the COHP method to investigate the chemical bonds in the three monolayers. For $\alpha-\mathrm{Sb}_{2} \mathrm{Te}_{2} \mathrm{Se}$ monolayer shown in Fig. S3(a), The CBM for $\alpha-\mathrm{Sb}_{2} \mathrm{Te}_{2} \mathrm{Se}$ is composed of anti-bonding states between $\mathrm{Sb}$ and Te atoms, and the VBM is composed of anti-bonding states between $\mathrm{Sb}$ and Te atoms, and between $\mathrm{Sb}$ and $\mathrm{Se}$ atoms. 


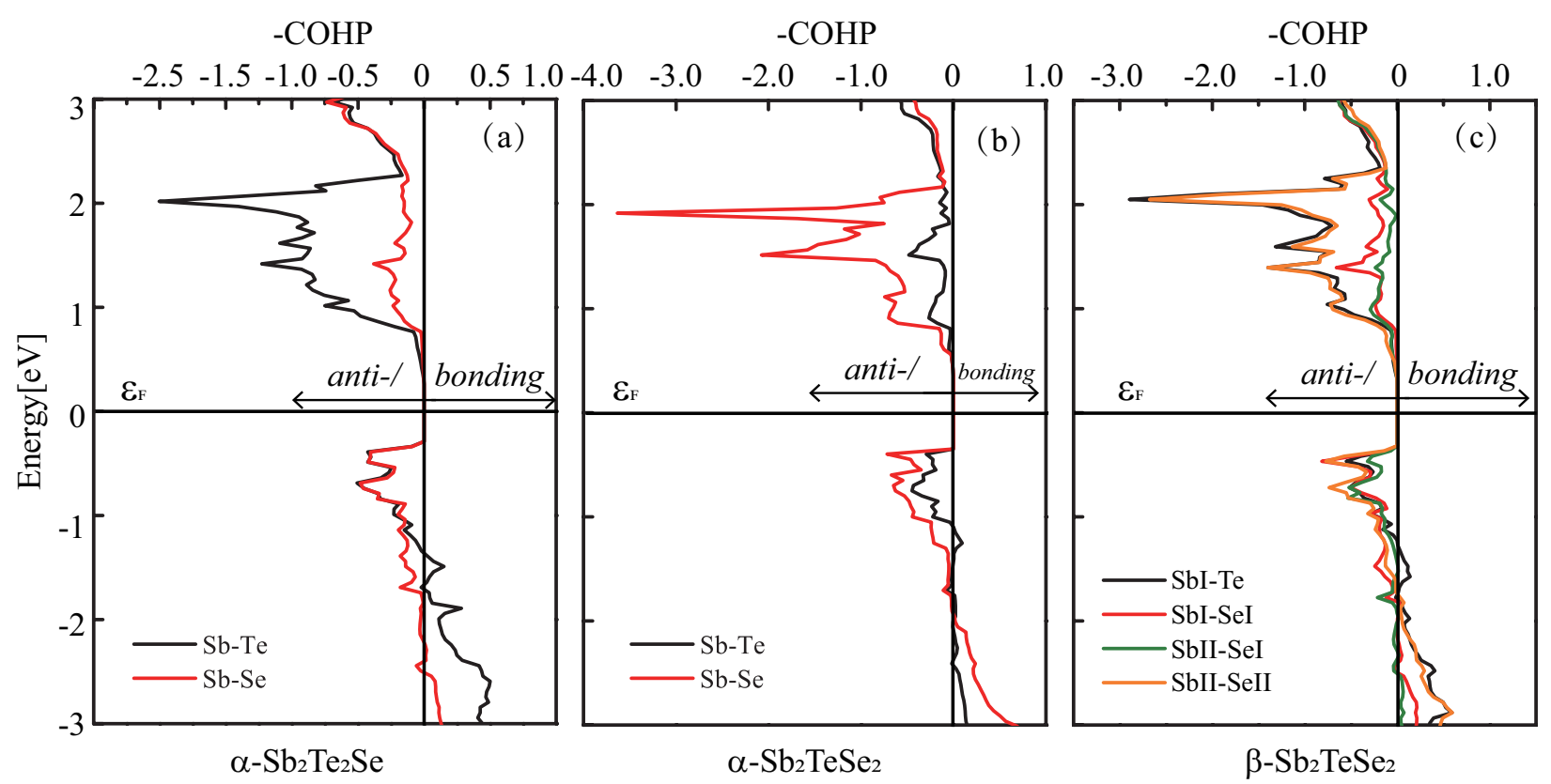

Figure S3: Energy-resolved COHP for (a) $\alpha-\mathrm{Sb}_{2} \mathrm{Te}_{2} \mathrm{Se},(\mathrm{b}) \alpha-\mathrm{Sb}_{2} \mathrm{TeSe}_{2}$ and (c) $\beta-\mathrm{Sb}_{2} \mathrm{TeSe}_{2}$.

For $\alpha-\mathrm{Sb}_{2} \mathrm{TeSe}_{2}$ monolayer shown in Fig. S3(b), The CBM for $\alpha-\mathrm{Sb}_{2} \mathrm{TeSe}_{2}$ is composed of anti-bonding states between $\mathrm{Sb}$ and Se atoms, and the VBM is composed of anti-bonding states between $\mathrm{Sb}$ and Te atoms, and between $\mathrm{Sb}$ and Se atoms. As shown in Fig. S3(c), for $\beta-\mathrm{Sb}_{2} \mathrm{TeSe}_{2}$, the CBM is composed of anti-bonding states between $\mathrm{Sb}$ and Te atoms, and between Sb- $p$ and Se- $p$ atoms; the VBM is composed by anti-bonding states between Sb and Te atoms, and between $\mathrm{Sb}$ and Se atoms.

We have calculated the charge density by considering the spin-orbit coupling (SOC) effects, as shown in Fig. S4. The SOC effects on charge densities is not significant.

\section{Thermal properties}

We calculate the Helmholtz free energy, entropy, and constant volume heat capacity of the three $\mathrm{Sb}_{2} \mathrm{Te}_{3-x} \mathrm{Se}_{x}$ monolayers, which are shown in Fig. S5(a,b,c). 


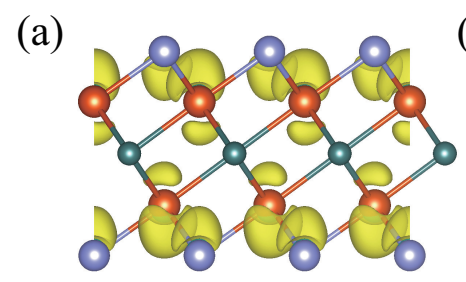

(b)

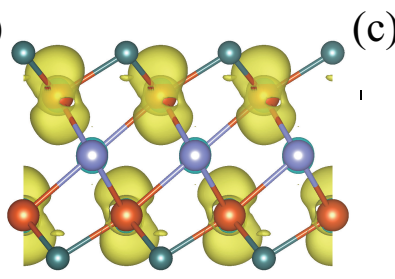

(c)
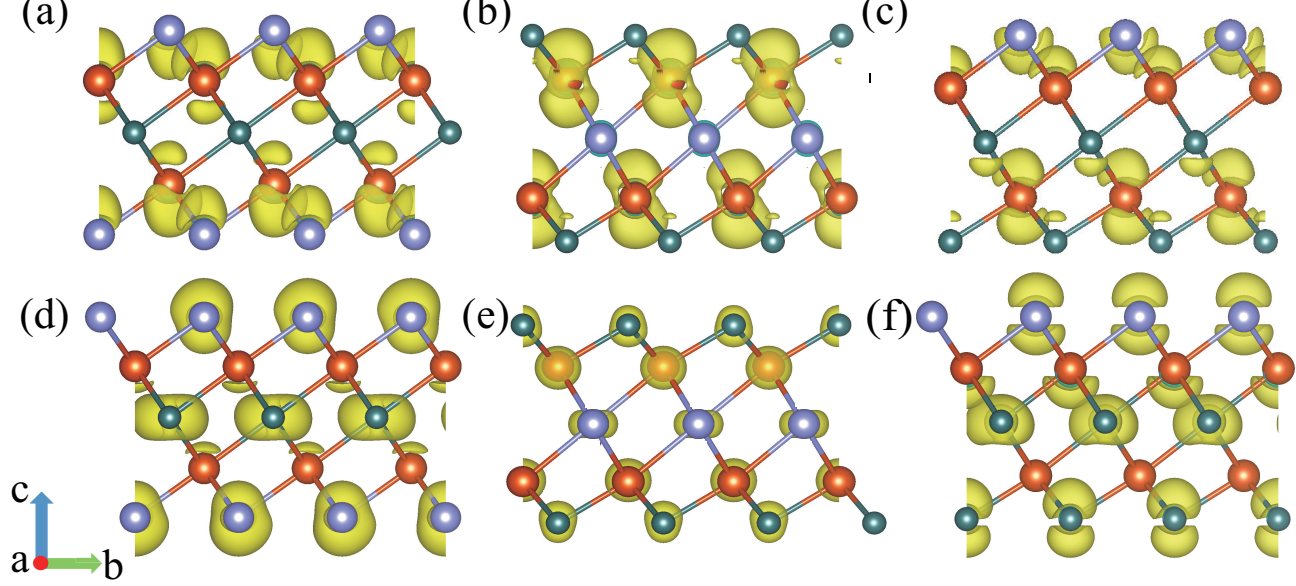

Figure S4: Iosurface plots of the charge density calculated by $\mathrm{PBE}+\mathrm{SOC}$. CBMs of (a) $\alpha$ $\mathrm{Sb}_{2} \mathrm{Te}_{2} \mathrm{Se},(\mathrm{b}) \alpha-\mathrm{Sb}_{2} \mathrm{TeSe}_{2}$ and (c) $\beta-\mathrm{Sb}_{2} \mathrm{TeSe}_{2}$. VBMs of (d) $\alpha-\mathrm{Sb}_{2} \mathrm{Te}_{2} \mathrm{Se},(\mathrm{e}) \alpha-\mathrm{Sb}_{2} \mathrm{TeSe}_{2}$ and (f) $\beta-\mathrm{Sb}_{2} \mathrm{TeSe}_{2}$.
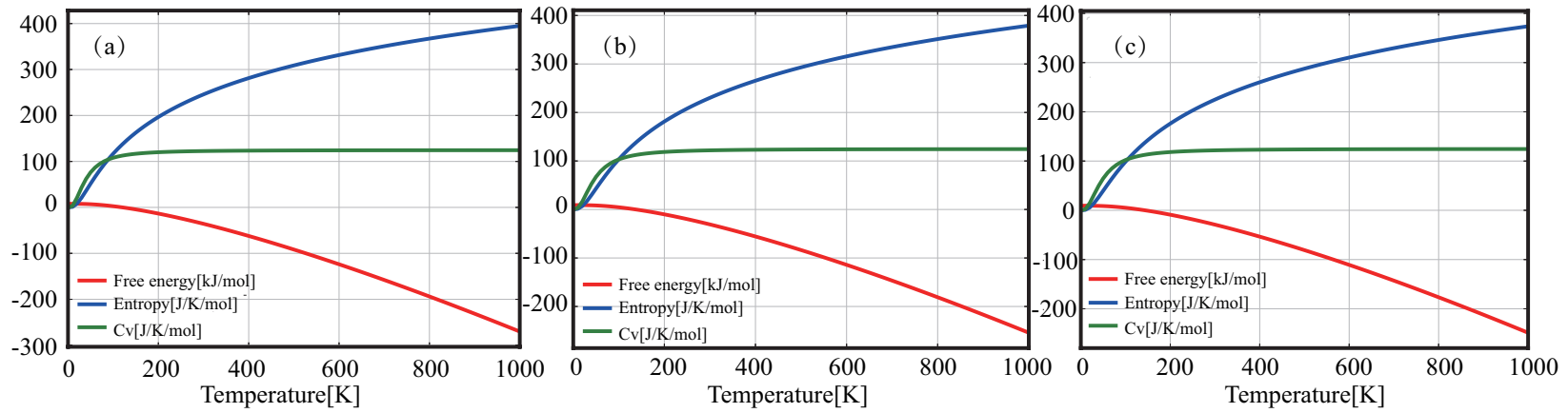

Figure S5: The thermal properties of (a) $\alpha-\mathrm{Sb}_{2} \mathrm{Te}_{2} \mathrm{Se},(\mathrm{b}) \alpha-\mathrm{Sb}_{2} \mathrm{TeSe}_{2}$ and (c) $\beta-\mathrm{Sb}_{2} \mathrm{TeSe}_{2}$. 


\section{Optical properties}

Fig. S6 shows the optical absorptions and photoconductivities for $\alpha-\mathrm{Sb}_{2} \mathrm{Te}_{2} \mathrm{Se} / \alpha-\mathrm{Sb}_{2} \mathrm{TeSe}_{2} / \beta$ $\mathrm{Sb}_{2} \mathrm{TeSe}_{2}$. The photoconductivities are calculated based on the maximally localized Wannier functions (MLWFs) method implemented in Wannier90, ${ }^{2}$ in which we use a total of 15 Wannier functions and the k-mesh of $125 \times 125 \times 1$.
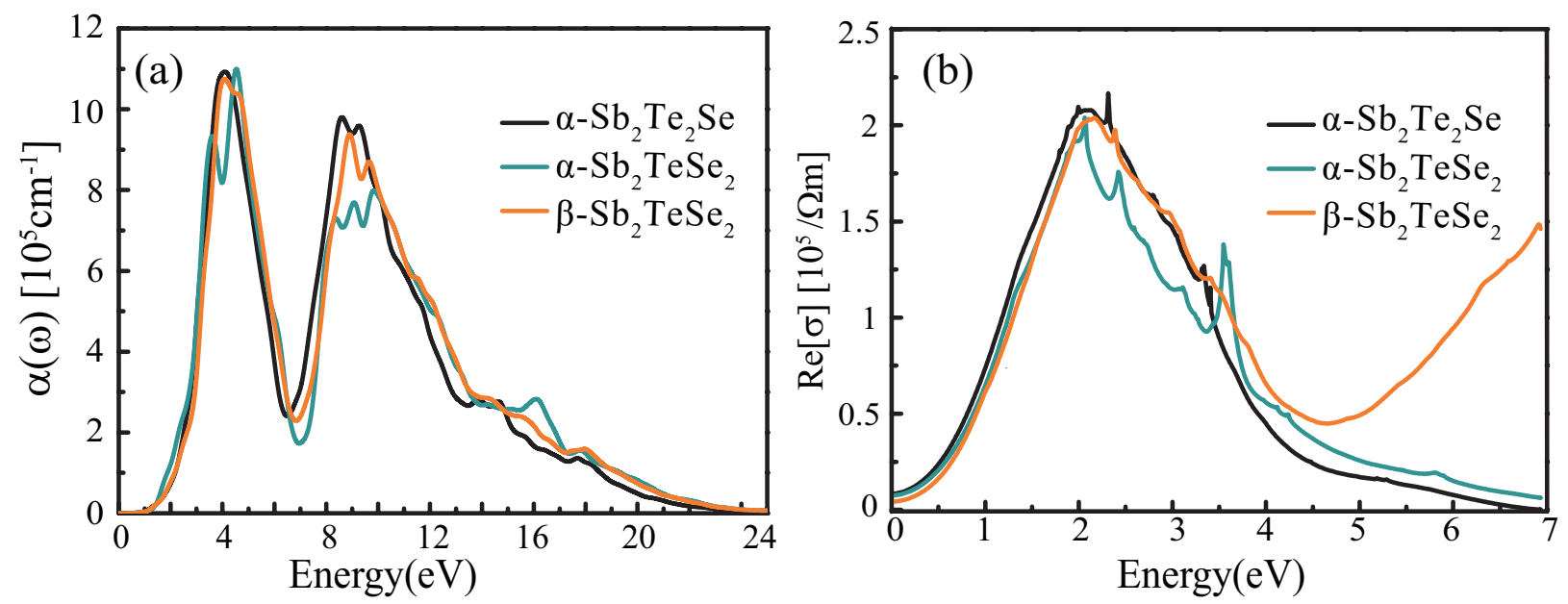

Figure S6: (a) Optical absorptions for $\alpha-\mathrm{Sb}_{2} \mathrm{Te}_{2} \mathrm{Se} / \alpha-\mathrm{Sb}_{2} \mathrm{TeSe}_{2} / \beta-\mathrm{Sb}_{2} \mathrm{TeSe}_{2}$. (b) Photoconductivities for $\alpha-\mathrm{Sb}_{2} \mathrm{Te}_{2} \mathrm{Se} / \alpha-\mathrm{Sb}_{2} \mathrm{TeSe}_{2} / \beta-\mathrm{Sb}_{2} \mathrm{TeSe}_{2}$.

The optical absorptions calculated by PBE without (with) SOC are shown in Fig. S7, which indicate the SOC effects on absorption coefficients are not significant.
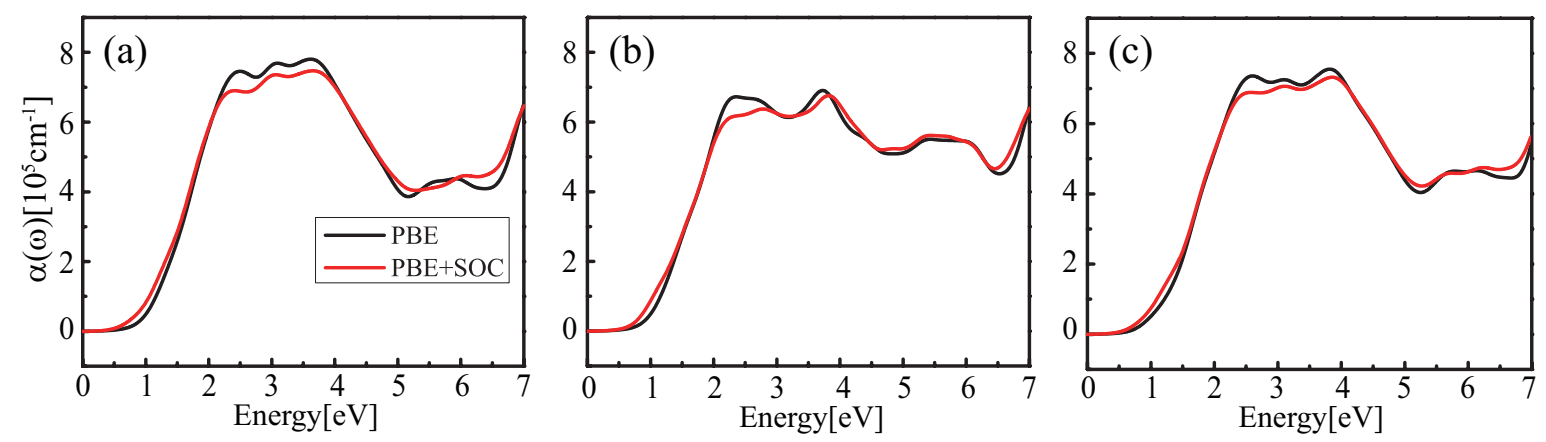

Figure S7: Absorption coefficients of $\alpha-\mathrm{Sb}_{2} \mathrm{Te}_{2} \mathrm{Se} / \alpha-\mathrm{Sb}_{2} \mathrm{TeSe}_{2} / \beta-\mathrm{Sb}_{2} \mathrm{TeSe}_{2}$ calculated by PBE and $\mathrm{PBE}+\mathrm{SOC}$. 


\section{$5 \quad$ Effective masses and mobilities}

We have calculated the angle-dependent effective masses of carriers by considering the spinorbit coupling (SOC) effects, as shown in Fig. S8. The angular distribution for the electron effective mass of CBM for $\beta-\mathrm{Sb}_{2} \mathrm{TeSe}_{2}$ as shown in Fig. S8(c) changes significantly, since the shifted CBM position located along $\Gamma$-M possesses $\sigma_{v}$ symmetry, which is different from the $C_{3 v}$ symmetry at $\Gamma$ point. Furthermore, the value for electron effective mass $m_{e}^{*}$ increases from about $0.1 m_{0}$ to about $0.2 / 0.3 m_{0}$, as shown in Table $\mathrm{S} 1$. The effective mass distribution of holes in $\alpha-\mathrm{Sb}_{2} \mathrm{TeSe}_{2}$ also changes because VBM changes from a point along $\Gamma-\mathrm{K}$ with $\mathrm{C}_{2}$ symmetry to a point along $\Gamma-\mathrm{M}$ with $\sigma_{d}$ symmetry, as shown Fig. S8(e).

To further investigate the SOC effects on carrier mobilities, we also calculate the mobilities for the three monolayers considering SOC effects, and the results are shown in Table S1. The mobilities considering SOC effects for $\alpha-\mathrm{Sb}_{2} \mathrm{Te}_{2} \mathrm{Se}$ and $\alpha-\mathrm{Sb}_{2} \mathrm{TeSe}_{2}$ are larger than those without SOC. For $\beta$-Sb $\mathrm{TeSe}_{2}$, since the effective masses of electrons along the $x$ and $y$ directions increase by more than twice, the mobilities along $x / y$ directions are reduced to $156 / 255 \mathrm{~cm}^{2} / \mathrm{Vs}$, which are much smaller than those without SOC effects.

Table S1: The effective masses, elastic modulus, deformation potential constants and mobilities for $\mathrm{Sb}_{2} \mathrm{Te}_{3-x} \mathrm{Se}_{x}$ monolayers with SOC effects.

\begin{tabular}{lcccccccc}
\hline & Direction & $m_{h}^{*}\left(m_{0}\right)$ & $m_{e}^{*}\left(m_{0}\right)$ & $\mathrm{C}(\mathrm{N} \cdot \mathrm{m})$ & $D_{l}^{h}$ & $D_{l}^{e}$ & $\mu_{h}\left(\mathrm{~cm}^{2} / V s\right)$ & $\mu_{e}\left(\mathrm{~cm}^{2} / V s\right)$ \\
\hline$\alpha-\mathrm{Sb}_{2} \mathrm{Te}_{2} \mathrm{Se}$ & $x$ & 0.214 & 0.089 & 107.066 & 5.634 & 10.788 & 1045 & 1648 \\
$\alpha-\mathrm{Sb}_{2} \mathrm{Te}_{2} \mathrm{Se}$ & $y$ & 0.408 & 0.089 & 114.482 & 6.979 & 10.800 & 200 & 1759 \\
$\alpha-\mathrm{Sb}_{2} \mathrm{TeSe}_{2}$ & $x$ & 0.433 & 0.106 & 105.232 & 5.835 & 9.242 & 234 & 1556 \\
$\alpha-\mathrm{Sb}_{2} \mathrm{TeSe}_{2}$ & $y$ & 2.401 & 0.106 & 100.438 & 6.688 & 9.271 & 5.5 & 1476 \\
$\beta-\mathrm{Sb}_{2} \mathrm{TeSe}_{2}$ & $x$ & 0.339 & 0.305 & 111.120 & 5.650 & 10.434 & 430 & 156 \\
$\beta-\mathrm{Sb}_{2} \mathrm{TeSe}_{2}$ & $y$ & 1.322 & 0.236 & 112.358 & 6.713 & 10.586 & 20 & 255 \\
\hline
\end{tabular}


(a)

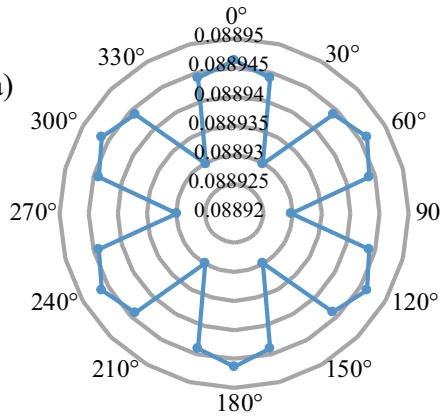

(d)

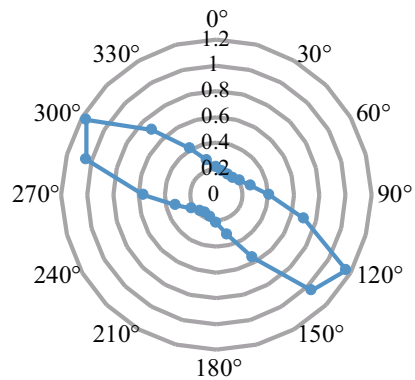

(b)

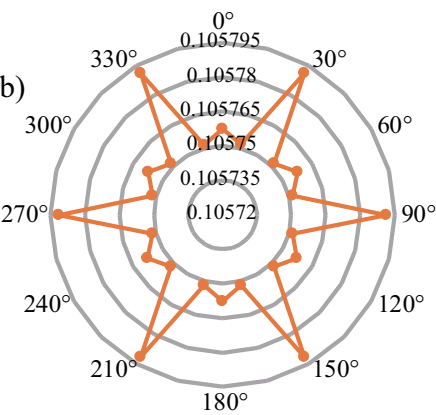

(e)

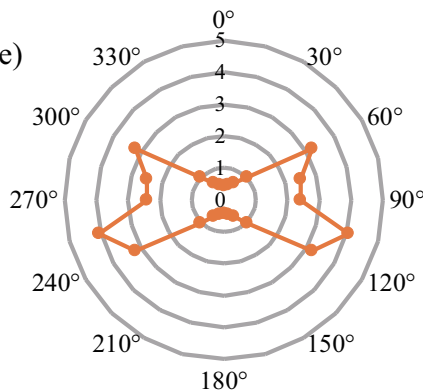

(c)

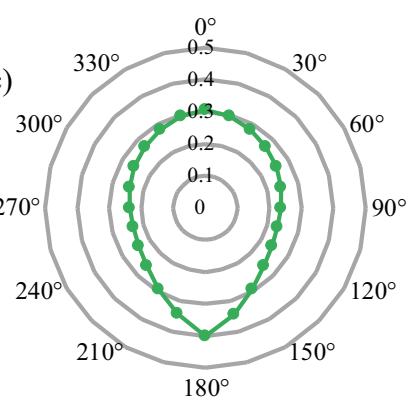

(f)

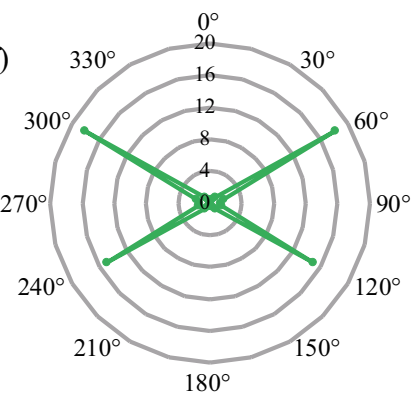

Figure S8: The carriers masses (units of $m_{0}$ ) of (a) electrons in $\alpha-\mathrm{Sb}_{2} \mathrm{Te}_{2} \mathrm{Se}$, (b) electrons in $\alpha$-Sb $\mathrm{SeSe}_{2},(\mathrm{c})$ electrons in $\beta-\mathrm{Sb}_{2} \mathrm{TeSe}_{2},(\mathrm{~d})$ holes in $\alpha-\mathrm{Sb}_{2} \mathrm{Te}_{2} \mathrm{Se},(\mathrm{e})$ holes in $\alpha-\mathrm{Sb}_{2} \mathrm{TeSe}_{2},(\mathrm{f})$ holes in $\beta-\mathrm{Sb}_{2} \mathrm{TeSe}_{2}$ calculated by $\mathrm{PBE}+\mathrm{SOC}$.

\section{$6 \quad$ Thermoelectric properties}

\subsection{Computational details}

We use the BoltzTraP code based on the rigid band approach to calculate the transport coefficients for the three kinds of $\mathrm{Sb}_{2} \mathrm{Te}_{3-x} \mathrm{Se}_{x}$ monolayers. ${ }^{3}$ The electrnoic transport properties, including carrier concentrations for holes and electrons $n_{h / e}$, electronic conductivity $\sigma$, electronic thermal conductivities $\kappa_{e}$ and Seebeck coefficient $S$ are obtained by solving the semiclassical Boltzmann transport equation (BTE) written as, ${ }^{3}$

$$
\begin{gathered}
n_{\mathrm{h}}(T, \mu)=\frac{2}{\Omega} \iint_{\mathrm{BZ}}\left[1-f_{0}(T, \varepsilon, \mu)\right] D(\varepsilon) \mathrm{d} \varepsilon \\
n_{\mathrm{e}}(T, \mu)=\frac{2}{\Omega} \iint_{\mathrm{BZ}} f_{0}(T, \varepsilon, \mu) D(\varepsilon) \mathrm{d} \varepsilon .
\end{gathered}
$$




$$
\begin{gathered}
\sigma_{\alpha \beta}(T, \mu)=\frac{1}{\Omega} \int \bar{\sigma}_{\alpha \beta}(\varepsilon)\left[-\frac{\partial f_{0}(T, \varepsilon, \mu)}{\partial \varepsilon}\right] d \varepsilon \\
\kappa_{\alpha \beta}^{e l}(T, \mu)=\frac{1}{e^{2} T \Omega} \int \bar{\sigma}_{\alpha \beta}(\varepsilon)(\varepsilon-\mu)^{2}\left[-\frac{\partial f_{0}(T, \varepsilon, \mu)}{\partial \varepsilon}\right] d \varepsilon \\
S_{\alpha \beta}(T, \mu)=\frac{1}{e T \Omega \sigma_{\alpha \beta}(T, \mu)} \int \bar{\sigma}_{\alpha \beta}(\varepsilon)(\varepsilon-\mu)\left[-\frac{\partial f_{0}(T, \varepsilon, \mu)}{\partial \varepsilon}\right] d \varepsilon,
\end{gathered}
$$

where $\Omega$ is the volume of the unit cell, $f_{0}$ is the Fermi-Dirac distribution, $\mu$ is the chemical potential, $D(\varepsilon)$ is the density of states, $\bar{\sigma}_{\alpha \beta}(\varepsilon)$ is the energy dependent conductivity tensor obtained by $\bar{\sigma}_{\alpha \beta}(\varepsilon)=\frac{1}{N} \sum_{i, \mathbf{k}} \bar{\sigma}_{\alpha \beta}(i, \mathbf{k}) \frac{\delta\left(\varepsilon-\varepsilon_{i, k}\right)}{d \varepsilon}$, where $N$ is the number of sampled $\mathbf{k}$ points and $\bar{\sigma}_{\alpha \beta}(i, \mathbf{k})$ can be calculated based on the kinetic theory, i.e. $\bar{\sigma}_{\alpha \beta}(i, \mathbf{k})=e^{2} \tau_{i, \mathbf{k}} v_{\alpha}(i, \mathbf{k}) v_{\beta}(i, \mathbf{k})$. The velocity of carrier $v_{\alpha, \beta}(i, \mathbf{k})$ is defined by $v_{i}=\frac{1}{\hbar} \frac{\partial \varepsilon_{i, \mathbf{k}}}{\partial k_{i}}(i=\alpha, \beta)$. The relaxation time was treated as both energy and direction independent constant, but depends on band structure and temperature. ${ }^{4}$

We use hiPhive Python package to calculate the second and third force-constant matrix for the three kinds of $\mathrm{Sb}_{2} \mathrm{Te}_{3-x} \mathrm{Se}_{x}$ monolayers. ${ }^{5}$ For $\alpha-\mathrm{Sb}_{2} \mathrm{Te}_{2} \mathrm{Se} / \alpha-\mathrm{Sb}_{2} \mathrm{TeSe}_{2} / \beta-\mathrm{Sb}_{2} \mathrm{TeSe}_{2}$, $8 \times 8 \times 1 / 7 \times 7 \times 1 / 6 \times 6 \times 1$ supercells are used. The reference structures are generated by using an average displacement of $0.03 \AA$ and an minimum distance of $2.0 \AA$ for reference force calculations. In the DFT calculation to calculate reference forces, the plane wave cutoff kinetic energy is set to $500 \mathrm{eV}$ and the convergence thresholds are set to $10^{-8} \mathrm{eV} / \AA$. The cluster spaces for the second-/third-/fourth-order force constants for the $\alpha-\mathrm{Sb}_{2} \mathrm{Te}_{2} \mathrm{Se}, \alpha-\mathrm{Sb}_{2} \mathrm{TeSe}_{2}$ and $\beta-\mathrm{Sb}_{2} \mathrm{TeSe}_{2}$ are constructed using the cutoff of $14.7 \AA / 6 \AA / 2 \AA, 12.5 \AA / 6 \AA / 2 \AA$ and $11.5 \AA / 6 \AA / 2 \AA$, respectively. We have performed the convergence test for the cutoff we use here. The secondand third-order force constants are extracted by the standard least squares regression.

We calculate the lattice thermal conductivities $\kappa_{l}$ at $300 \mathrm{~K}$ under the relaxation-time approximation (RTA) and full Boltzmann transport equation (BTE) using almaBTE software package. ${ }^{6}$ 


\subsection{Numerical results}

The electronic thermal conductivity $\kappa_{e}$, electronic conductivity $\sigma$ and Seebeck coefficient $S$ at $300 \mathrm{~K}$ are calculated by BoltzTrap code. ${ }^{3}$ The calculated lattice thermal conductivities $\kappa_{l}$ of $\alpha-\mathrm{Sb}_{2} \mathrm{Te}_{2} \mathrm{Se} / \alpha-\mathrm{Sb}_{2} \mathrm{TeSe}_{2} / \beta-\mathrm{Sb}_{2} \mathrm{TeSe}_{2}$ are $1.79 / 0.83 / 1.23 \mathrm{~W} / \mathrm{mK}$, respectively. The calculated $\sigma, S$ and total thermal conductivity $\kappa$ obtained by summing $\kappa_{l}$ and $\kappa_{e}$ are shown in Fig. S9.
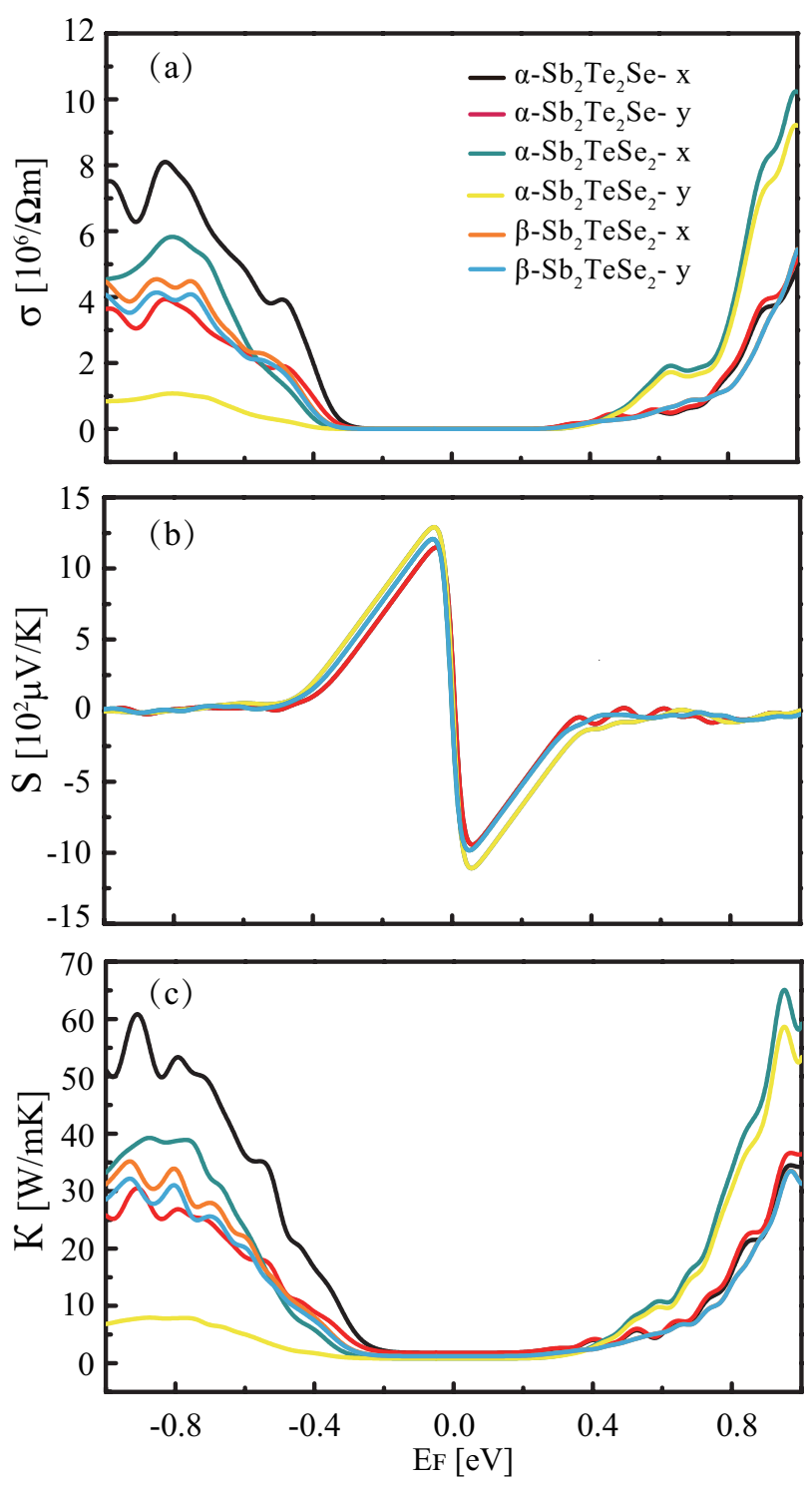

Figure S9: Electrical conductivity $\sigma$, Seebeck coefficient $S$ and total thermal conductivities $\kappa$ of $\alpha-\mathrm{Sb}_{2} \mathrm{Te}_{2} \mathrm{Se} / \alpha-\mathrm{Sb}_{2} \mathrm{TeSe}_{2} / \beta-\mathrm{Sb}_{2} \mathrm{TeSe}_{2}$ monolayers. 


\section{References}

(1) Dronskowski, R.; Bloechl, P. E. Crystal orbital Hamilton populations (COHP): energyresolved visualization of chemical bonding in solids based on density-functional calculations. The Journal of Physical Chemistry 1993, 97, 8617-8624.

(2) Mostofi, A. A.; Yates, J. R.; Pizzi, G.; Lee, Y.-S.; Souza, I.; Vanderbilt, D.; Marzari, N. An updated version of Wannier90: A tool for obtaining maximally-localised Wannier functions. Comput. Phys. Commun. 2014, 185, 2309-2310.

(3) Madsen, G. K. H.; Singh, D. J. BoltzTraP. A code for calculating band-structure dependent quantities. Computer Physics Communications 2006, 175, 67-71.

(4) Chen, C.-L.; Wang, H.; Chen, Y.-Y.; Day, T.; Snyder, G. J. Thermoelectric properties of p-type polycrystalline SnSe doped with Ag. J. Mater. Chem. A 2014, 2, 11171-11176.

(5) Eriksson, F.; Fransson, E.; Erhart, P. The Hiphive Package for the Extraction of HighOrder Force Constants by Machine Learning. Advanced Theory and Simulations 2019, 2, 1800184 .

(6) Carrete, J.; Vermeersch, B.; Katre, A.; van Roekeghem, A.; Wang, T.; Madsen, G. K.; Mingo, N. almaBTE : A solver of the space-time dependent Boltzmann transport equation for phonons in structured materials. Computer Physics Communications 2017, 220, $351-362$. 